\title{
A simple and reliable method to estimate platelet counts that will help in an emergency
}

\author{
Muthu $\mathbf{S}^{1}$, Shivashekar $\mathbf{G}^{2}$, Koshalya $\mathbf{R}^{3}$ \\ ${ }^{1}$ Dr. Muthu Sudalaimuthu, Assistant Professor, ${ }^{2}$ Dr. Shivashekar Ganapathy, Professor and Head, ${ }^{3}$ Dr. Koshalya \\ Rajendran, Post-graduate Student, Department of Pathology, SRM Medical College Hospital and Research Centre, \\ Kattankulathur, Kancheepuram District, Tamil Nadu, India.
}

Address for Correspondence: Dr. Muthu S, E-mail: drmuthus@ gmail.com

\begin{abstract}
Background: Platelet counts can decrease suddenly in various disorders which need to be diagnosed and managed as early as possible to prevent life-threatening bleeding. Although various methods to estimate platelet counts have been described in the literature, study on platelet count estimation from unstained peripheral blood smears has been mentioned only once in the literature, that too only recently. Considering the potential usefulness of this technique in an emergency, we aimed to estimate the platelet counts from unstained peripheral blood smears and compared it with results from stained smears.Materials and Methods: Platelet counts were estimated in 200 cases using unstained peripheral smears by observing them under oil immersion objective with a lowered condenser and closed iris diaphragm. Platelets were counted in ten oil immersion fields and their average was multiplied by 15000. The same smears were stained and platelet counts determined by routine method. Student's t test was used to compare the results of two methods. Results: Platelet counts obtained from unstained peripheral smears were not significantly different from stained smears ( $\mathrm{p}$ value of 0.243). Although platelet counts were underestimated in $75 \%$ cases, the maximum deviation was only 15000 in cases of thrombocytopenia and 30000 in patients with normal platelet counts. Conclusion: Platelet counts from unstained peripheral blood smears yield results similar to stained peripheral smears. It can be used in an emergency situation when the routine laboratory facilities are not available and when the treating clinician wants to gain early vital information.
\end{abstract}

Keywords: Hematology, Peripheral blood smear, Platelets, Platelet count

\section{Introduction}

Deficiency of platelets can result in life-threatening bleeding which can be prevented by prophylactic platelet transfusions [1]. Such a rapid reduction in platelet counts can occur in a variety of disorders and have to be diagnosed early so that appropriate management measures can be taken [2-7]. Various methods have been enumerated in the literature to estimate platelet counts which include hemocytometer, phase contrast microscopy, examination of stained peripheral blood smears, automated hematology analyzers and fluorescent labeling techniques [8-12]. Estimation of platelet counts from unstained peripheral smears has been mentioned only once in the literature, that too very recently [13]. Hence the aim of the study was to estimate the platelet counts from unstained peripheral blood smears and to compare them with the

Manuscript received: $10^{\text {th }}$ October 2016

Reviewed: $25^{\text {th }}$ October 2016

Author Corrected: $8^{\text {th }}$ November 2016

Accepted for Publication: $20^{\text {th }}$ November 2016 platelet counts from stained smears.

\section{Materials and Methods}

200 blood samples that were received in our laboratory for performing complete blood counts were included in our study after obtaining informed consent from the patients. Samples were collected in tubes containing Ethylene diamine Tetraacetic acid as anti-coagulant and thin peripheral blood smears made from them within three hours. Smears were air dried and examined using a microscope (Olympus) with field number 18. Smears were first viewed under 10X and 40X objective lenses. Smears with platelet clumps were excluded from the study. An area where the platelets were equally distributed was selected at the junction of body and tail where the red blood cells were in monolayer. This area was marked using a lead pencil. Condenser was lowered and iris diaphragm was closed to minimize the 
Research Article

illumination. The marked area was then viewed under oil immersion objective but without using any oil. Platelets were counted in ten fields and their average was taken. The same smears were stained with Leishman stain and platelets were counted under oil immersion objective for ten fields with the condenser up. Average number of platelets per field was calculated in this method also. Platelet counts were calculated in both the methods by multiplying the average number of platelets per field by 15000 . Platelet counts obtained from both the methods were compared by student's t test with Microsoft Excel 2010.

\section{Results}

Platelets were seen as small refractile bodies in unstained peripheral blood smears (Figure 1). Platelet count values from the stained smears ranged from 31500 to 609000 per microliter with a mean of 272535 whereas in the unstained smears it ranged from 30000 to 552000 with a mean of 260467.5. In most of the cases (150 cases-75\%), platelet count was underestimated in the unstained smears. Maximum difference was noted in cases of thrombocytosis where the deviation was upto 63000 below the value obtained from stained smears. In cases with normal count, this deviation was upto 30000 and in cases with thrombocytopenia maximum difference was only 15000.

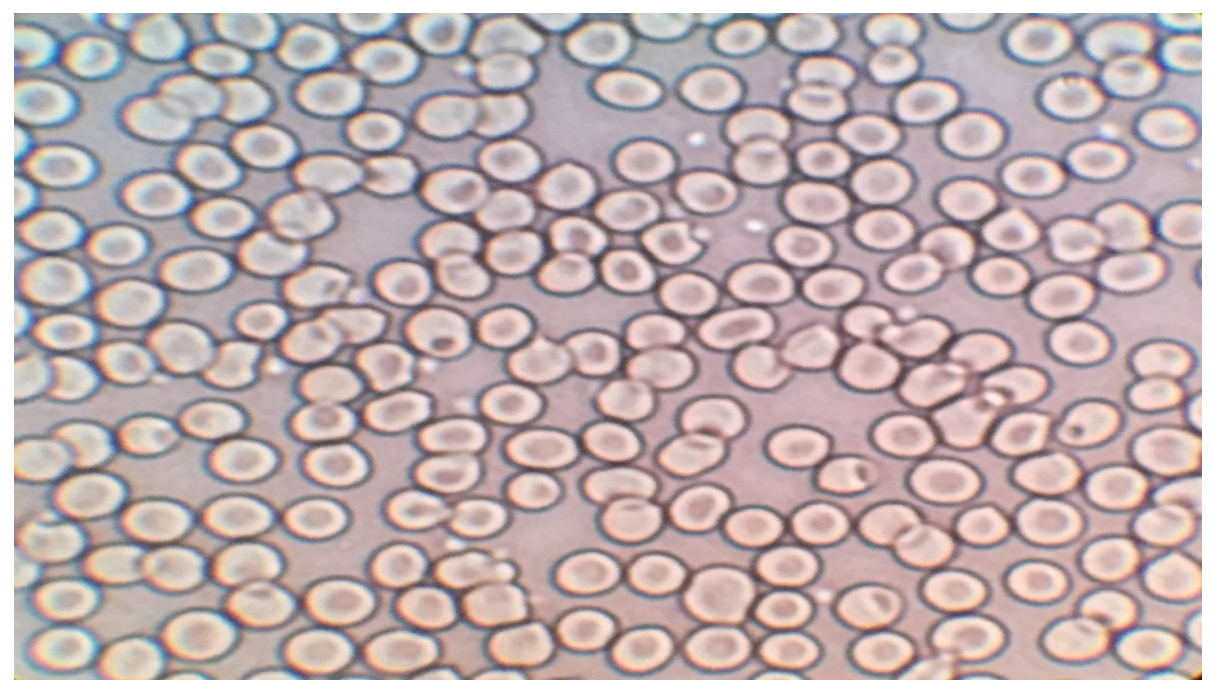

Figure-1: Unstained peripheral smear showing platelets as small refractile bodies scattered among the red blood cells $(\mathbf{X 1 0 0 0})$

In $20.5 \%$ of the cases, platelet count was overestimated in the stained smears. This was seen in all platelet ranges and the overestimation was maximum 12000 only. Both the methods had same platelet count in 9 cases (4.5\%). Results from the two methods did not show any statistically significant difference (p value of 0.243 ).

\section{Discussion}

Platelet counting is one of the areas in hematology where manual methods still have a significant role. This is because accuracy of automated hematology analyzers is low when it comes to platelet counts particularly in thrombocytopenic patients $[14,15]$. Various methods have been described in the literature to estimate platelet counts, including manual methods [8-12]. A manual method that was widely in use is the estimation of platelet count from stained peripheral blood films by counting the number of platelets in ten oil immersion fields [11,16]. Although this method has some drawbacks like inter-obsever variability, this method is used even today to verify the platelet count results of automated analyzers [17].
A huge advantage of this method is, this method does not depend on the automated analyzer values. Hence this method is useful in emergency situations when the automated analyzer facilities are not available round the clock. However difficulties may arise in this method when there is sub-optimal staining and stain artefacts. Also a round the clock laboratory service is not available in many rural areas even today. It is under these circumstances this technique will be of great help.

Our study results have shown that platelet counts from unstained peripheral smears are not significantly different from those obtained from stained smears. Although platelet counts were underestimated in most 
Research Article

of the cases, the difference was within 15000 in cases of thrombocytopenia and within 30000 in cases with normal platelet counts. These are acceptable differences considering that variations to this degree may be present even when the same stained smears are counted again by the same person $[11,18]$. Thus these differences might be because of the counting procedure and not just because these were unstained smears. Another reason why platelet counts were consistently underestimated could be that the smaller sized platelets were missed during counting in unstained peripheral smears [13].

Platelet levels were overestimated in unstained smears only in $20.5 \%$ of the cases and the maximum difference was only 12000 . This again is negligible considering that variations can be seen in this counting method as described above. Another reason for overestimation could be fragments of red and white blood cells being counted as platelets [13]. However we did not have much difficulty in differentiating platelets from these substances due to the refractile nature of the platelets. Thus overall the differences were very little particularly in samples in thrombocytopenic range.

All that this method needs is a microscope and slides. All that needs to be done is to make a peripheral smear and count these refractile platelets under the microscope. Since every undergraduate medical student is trained to make a peripheral smear and since this technique does not need any staining, we feel this method of platelet estimation can be done even by the treating clinician in an emergency, if they are a little trained. This can be very helpful in the rural setup when round the clock laboratory facilities are not available. Proper hematological workup and treatment measures can be done following that. The turnaround time for this procedure ranges from five to seven minutes. This is very short when compared to the time taken from routine sample collection to receiving reports even in an urban setup. Hence even in an urban setup this technique will be of great help as an emergency measure as it helps in planning the management early.

Last but not the least, even in hematology laboratories when there is some problem with the stains, doing a correct platelet count will be difficult. This technique can be used in such circumstances to crosscheck the platelet values from automated analyzers till the problems with the stain are corrected. However in spite of the simplicity of the procedure, short turnaround time and comparable degree of accuracy, we would not recommend this as a routine alternative to stained peripheral smears as many vital findings in the red blood cell and white blood cells could be missed in unstained peripheral smears. We would recommend this technique only as a backup tool when the staining procedure is not available or suboptimal and as a preliminary tool by the treating clinician to gain early vital information.

Certain precautions need to be taken while using this method of platelet estimation. Eyepieces and objective lens should be properly cleaned before counting the platelets as it can lead to artefacts which will interfere with platelet counting. Artefacts from eyepiece and objective lenses will be seen in all the fields at the same position in contrast to the platelets. When there is a doubt, adjusting the fine adjustment screw a little will help in identifying the platelets by their refractile nature. Smears should be viewed only after it has got dried and care should be taken so that the cells do not get stuck to the oil immersion objective lens while viewing them.

\section{Conclusion}

To conclude, platelet counts from unstained peripheral blood smears yield results similar to stained peripheral smears. This is a very simple and reliable technique. It can be used in an emergency situation when the routine laboratory facilities are not available (particularly in rural areas) and when the clinician wants to gain early vital information. This method is cost-effective and has a very short turnaround time.

Acknowledgements- We thank all the technicians of our hematology laboratory for their technical helps during the study.

Funding: Nil, Conflict of interest: None initiated, Permission from IRB: Yes

\section{References}

1. Kumar A, Mhaskar R, Grossman BJ, Kaufman RM, Tobian AAR, Kleinman S, et al. Platelet transfusion: a systematic review of the clinical evidence. Transfusion. 2015 May;55(5):1116-1127.

2. Dusse LM, Alpoim PN, Silva JT, Rios DRA, Brandão AH, Cabral ACV. Revisiting HELLP syndrome. Clin Chim Acta. 2015 Dec 7; 451(Pt B): 117-20.

3. Szokol JW. Heparin-induced thrombocytopenia. Semin Cardiothorac Vasc Anesth. 2010Mar;14(1):73-4. 
Research Article

4. Scully M, Thomas M, Underwood M, Watson H, Langley K, Camilleri RS, et al. Thrombotic thrombocytopenic purpura and pregnancy: presentation, management, and subsequent pregnancy outcomes. Blood. 2014 Jul 10;124(2):211-9.

5. Cines DB, Liebman H, Stasi R. Pathobiology of secondary immune thrombocytopenia. Semin Hematol. 2009 Jan;46(1 Suppl 2):S2-14.

6. Tong SF, Aziz NA, Chin GL. Predictive value of thrombocytopaenia in the diagnosis of dengue infection in outpatient settings. Med J Malaysia. 2007 Dec;62 (5):390-3.

7. Rahman M, Ortega-Lopez A, Powers A. Sudden Development of Thrombocytopenia After Reversal of Anticoagulation for Surgery. Lab Med. 2016 Feb;47 (1):48-51.

8. Brecher G, Cronkite EP. Morphology and enumeration of human blood platelets. J Appl Physiol. 1950 Dec;3(6):365-77.

9. Stavem P. Platelet count by phase contrast microscopy--new diluting fluid for better visualization. Scand J Clin Lab Invest. 1974 Apr;33(2):121-3.

10. Briggs $\mathrm{C}$, Harrison $\mathrm{P}$, Machin SJ. Continuing developments with the automated platelet count. Int $\mathbf{J}$ Lab Hematol. 2007 Apr;29(2):77-91.

11. Nosanchuk JS, Chang J, Bennett JM. The analytic basis for the use of platelet estimates from peripheral blood smears. Laboratory and clinical applications. Am J Clin Pathol. 1978 Apr;69(4):383-7.
12. International Council for Standardization in Haematology Expert Panel on Cytometry, International Society of Laboratory Hematology Task Force on Platelet Counting. Platelet counting by the RBC/platelet ratio method. A reference method. Am J Clin Pathol. 2001 Mar;115(3):460-4.

13. Umashankar T, Thomas BM, Sahana P. Estimation of platelet count in unstained peripheral blood smears in comparison with stained smears and evaluation of its efficacy. Malays J Pathol. 2014 Dec;36(3):195-9.

14. De la Salle BJ, McTaggart PN, Briggs C, Harrison P, Doré CJ, Longair I, et al. The accuracy of platelet counting in thrombocytopenic blood samples distributed by the UK National External Quality Assessment Scheme for General Haematology. Am J Clin Pathol. 2012 Jan;137(1):65-74.

15. Oliveira RAG, Takadachi MM, Nonoyama K, Barretto OC de O. Is automated platelet counting still a problem in thrombocytopenic blood? Sao Paulo Med J. 2003 Jan 2;121(1):19-23.

16. Webb DI, Parker L, Webb K. Platelet count assessment from peripheral blood smear (PBS). Alaska Med. 2004 Dec;46(4):92-5.

17. Bajpai R, Rajak C, Poonia M. Platelet estimation by peripheral smear: Reliable, rapid, cost effective method to assess degree of thrombocytopenia. International Journal of Medical Science Research and Practice 2015;2(2):90-93.

18. Gao Y, Mansoor A, Wood B, Nelson H, Higa D, Naugler C. Platelet count estimation using the CellaVision DM96 system. J Pathol Inform. 2013;4:16.

\section{How to cite this article?}

Muthu S, Shivashekar G, Koshalya R. A simple and reliable method to estimate platelet counts that will help in an emergency.Trop J Path Micro 2016;2(3):142-145.doi: 10.17511/jopm.2016.i03.10 